\title{
Hyperglycemia induces endoplasmic reticulum stress-dependent CHOP expression in osteoblasts
}

\author{
WEI LIU ${ }^{1}$, XIAOQING ZHU ${ }^{2}$, QIAN WANG ${ }^{2}$ and LINLIN WANG ${ }^{2}$ \\ ${ }^{1}$ Department of Prosthetics, Stomatology Hospital, School of Medicine, Zhejiang University; \\ ${ }^{2}$ Department of Basic Medical, School of Medicine, Zhejiang University, Hangzhou 310058, P.R. China
}

Received January 5, 2013; Accepted February 19, 2013

DOI: $10.3892 /$ etm.2013.978

\begin{abstract}
Diabetic osteoporosis is a metabolic bone disease responsible for global health problems. Hyperglycemia induces osteopenia, increases bone fragility and unbalances the coupling of osteoblasts and osteoclasts. The mechanism is, however, unknown. For the purpose of this study, we hypothesized that hyperglycemia destroys endoplasmic reticulum (ER) homeostasis, activates C/EBP-homologous protein (CHOP) and induces osteoblast apoptosis under diabetic conditions. Diabetic rats were created by injecting streptozotocin (STZ) $65 \mathrm{mg} / \mathrm{kg}$ intraperitoneally and their osteoblasts were cultured under high-glucose medium in vitro. The bone mineral density (BMD) and pathological changes of the rats' femurs were observed. The expression of CHOP in osteoblasts was assayed using immunohistochemistry and western blot analysis. Six weeks after diabetic model establishment, a significant decrease was found in the BMD of the diabetic rat femurs, and the numbers of osteoblasts under cortical bone were also reduced. The expression of the ER stress regulator $\mathrm{CHOP}$ in osteoblasts of diabetic rats or high-glucose medium was also elevated $(\mathrm{P}<0.01)$. The present results demonstrated that hyperglycemia elevated the expression of $\mathrm{CHOP}$ and finally led to osteoporosis. This suggested that elevating the expression of CHOP may play a role in diabetic osteoporosis.
\end{abstract}

\section{Introduction}

Patients with diabetes appear to have a higher risk of hip and upper extremity fracture compared with non-diabetics (1). Diabetes mellitus (DM) influences bone metabolism and contributes to bone loss (2). A histomorphometric study in DM has described low recruitment of osteoblasts and diminished

Correspondence to: Dr Linlin Wang, Department of Basic Medical, School of Medicine, Zhejiang University, Yuhangtang Road 866\#, Hangzhou 310058, P.R. China

E-mail: wanglinlin@zju.edu.cn

Key words: diabetic osteoporosis, C/EBP-homologous protein, endoplasmic reticulum stress, osteoblasts mineral apposition rates with no mineralization defects (3). Animal studies have also demonstrated that bone formation was impaired in a diabetic mouse model during tibial distraction osteogenesis (4). In a bone-loss mouse model, DM also decreased osteoclastogenesis, reduced bone formation and enhanced osteoblast apoptosis (5). The mechanism of DM-related osteoporosis has yet to be elucidated.

Under hyperglycemia, the unbalanced coupling of bone resorption and formation in remodeling promotes excess bone resorption (6). Thus, DM causes a more persistent response, greater loss of attachment and more alveolar bone resorption and impaired new bone formation. The key mechanism of osteoblast-osteoclast coupling imbalance is the regulation of osteoblast cellular apoptosis (7). Under normal conditions, stress within the ER triggers an adaptive cellular mechanism known as the unfolded protein response (UPR) that attempts to return the cell to homeostasis under hyperglycemia. If hyperglycemia persists and cellular homeostasis is not restored, the ER stress response initiates cell death stimuli that lead to ER stress-induced apoptosis (8). Initiation of ER stress-induced apoptosis through ER stress response signaling involves transcriptional activation of the C/EBP-homologous protein (CHOP) $(9,10)$. CHOP is induced in response to cellular stress, especially ER stress, and is involved in the ER stress-induced apoptosis pathway (11). In this study, it was hypothesized that hyperglycemia may induce CHOP-mediated ER stress apoptosis in osteoblasts and result in unbalanced coupling of osteoblasts and osteoclasts and ultimately lead to diabetic osteoporosis.

The aim of the present study was to explore the pathological changes and the $\mathrm{CHOP}$ activity in diabetic rats femurs and high-glucose cultured osteoblasts.

\section{Materials and methods}

Animal model induction and bone mineral density (BMD) observation. Twenty male Sprague Dawley (SD) rats (purchased from the Experiment Animal Center of Zhejiang University) were divided into two groups $(n=10)$ at random. The rats in the diabetic group were fasted for $10 \mathrm{~h}$ and injected intraperitoneally with streptozotocin (STZ) (Alexis Corporation, Switzerland) $65 \mathrm{mg} / \mathrm{kg}$ to induce diabetes. The other 10 rats formed the control group and they were fasted 
for $10 \mathrm{~h}$ and injected with $0.9 \%$ saline. The rats with blood glucose $>16 \mathrm{mmol} / \mathrm{l} 48 \mathrm{~h}$ after injection were considered diabetic. All procedures were approved by the Zhejiang University Institutional Animal Care and Use Committee.

Six weeks after diabetes was induced, all rats were anesthetized, their thoracic cavities opened and perfused intracardially with normal saline. Following saline perfusion, the animals were perfused with $300-400 \mathrm{ml}$ fixative containing $4 \%$ paraformaldehyde in $0.1 \mathrm{~mol} / \mathrm{l}$ phosphate buffer $(\mathrm{pH}$ 7.4). After perfusion, both femurs were extracted. The BMD of the left femurs was determined using a small animal radiophotography system (Faxitron model MX20; Tucson, AZ, USA). The right femurs were used for hematoxylin and eosin (H\&E) staining and immunohistochemistry assay.

Osteoblast culture and western blot analysis. Primary osteoblasts were derived from newborn rat calvaria as previously described (24). Cells were seeded in 6-, 24- or 96-well plates in Dulbecco's modified Eagle's medium (DMEM; Invitrogen, Carlsbad, CA, USA) supplemented with antibiotics (penicillin $100 \mathrm{U} / \mathrm{ml}$ and streptomycin $100 \mu \mathrm{g} / \mathrm{ml}$ ) and $15 \%$ fetal bovine serum (FBS) and incubated at $37^{\circ} \mathrm{C}$ in a humidified atmosphere of $5 \% \mathrm{CO}_{2}$ and $95 \%$ air. Cells were used between the second and the fourth passages. The third passage cells were divided into a normal medium group and a high-glucose medium group (concentrations of glucose $100 \mathrm{mmol} / \mathrm{l}$ ). The osteoblasts of both groups were cultured for $48 \mathrm{~h}$ and washed twice with phosphate-buffered saline (PBS). Ten wells of osteoblasts for each group were used for CHOP western blot analysis.

The cells were prepared in lysis buffer and centrifuged $\left(12,000 \mathrm{x} \mathrm{g}\right.$ for $10 \mathrm{~min}$ at $\left.4^{\circ} \mathrm{C}\right)$ to remove cellular debris. The protein concentration was determined by the Lowry method using a Bio-Rad DC protein assay kit (Bio-Rad, Hercules, CA, USA). The lysates containing equal amounts of protein $(50 \mu \mathrm{g})$ were resolved using 8-10\% SDS-polyacrylamide gel electrophoresis and transferred onto Millipore (Billerica, MA, USA) nitrocellulose membranes. The reactions were stopped with a solution containing 5\% skimmed milk in Tris-buffered saline with $0.05 \%$ Tween-20 (TBST) for $1 \mathrm{~h}$ at room temperature and treated with CHOP antibodies (1:2,000, Santa Cruz Biotechnology Inc., Santa Cruz, CA, USA) in TBST overnight at $4^{\circ} \mathrm{C}$, washed for $1 \mathrm{~h}$ with TBST and further probed with secondary horseradish peroxidase (HRP; 1:2,000) in TBST for $1 \mathrm{~h}$ at room temperature. The immune complexes were visualized using an enhanced chemiluminescence (ECL) detection system according to the manufacturer's protocols. The densitometric analysis of the bands was assayed using Quantity One (Bio-Rad).

$H \& E$ staining and immunohistochemistry assay. The remaining right femurs were fixed in the same fixative for $4 \mathrm{~h}$ and placed in $30 \%$ phosphate-buffered sucrose until the tissue sank. Sections $12 \mu \mathrm{m}$ thick were cut on a freezing microtome through the coronary planes of the proximal femur for H\&E staining and diaminobenzidine (DAB) immunohistochemical staining.

Femur sections were rinsed in $0.01 \mathrm{M}$ PBS and mounted onto $0.02 \%$ poly-1-lysine-coated slides. The ABC system was used with $\mathrm{DAB}$ as the chromagen. Tissue sections were first washed in PBS and incubated in $1 \%$ bovine serum albumin
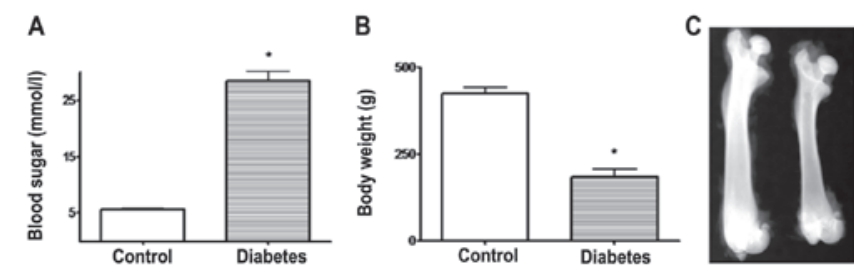

Figure 1. Body weight, blood glucose and proximal femoral bone mineral density (BMD) analysis in the two groups six weeks after streptozotocin (STZ) injection. (A) Blood glucose in the two groups; (B) Body weight in the two groups. $\mathrm{P}<0.01$, vs. control. (C) $\mathrm{X}$-ray images of a left femur isolated from the two groups. Left, control rat; Right, diabetic rat showed more bone loss and reduced length.

(BSA) for $30 \mathrm{~min}$. Tissues were incubated overnight at $4^{\circ} \mathrm{C}$ in the medium of PBS with CHOP antibody (1:100) plus $1 \%$ BSA. Control sections were incubated in PBS and $1 \%$ BSA. The following day, the sections were incubated in a biotinylated goat-anti-mouse secondary antibody (diluted 1:200 in PBS, Boster Biotechnology Company, Wuhan, China) and subsequently in an avidin-HRP solution. Immunolabeling was visualized with $0.05 \%$ DAB plus $0.3 \% \mathrm{H}_{2} \mathrm{O}_{2}$ in PBS. The sections were dehydrated through ethanol and xylene before mounting.

Immunohistochemistry results were analyzed using CHOP-positive osteoblast in femur per $\mathrm{mm}^{2}$ of two groups of rats under a Nikon microscope (Nikon E600, Nikon Company, Japan) at final magnifications of $\mathrm{x} 400$.

Statistical analysis. CHOP-positive cells in each visual field under the microscope were counted at x200 magnification. The data represent the means $\pm \mathrm{SD}$. The differences were evaluated by analysis of two-tailed t-tests. $\mathrm{P}<0.05$ was considered to indicate a statistically significant result. All computations were performed using SPSS 15.0 (SPSS Inc., Chicago, IL, USA).

\section{Results}

Body weight, blood glucose and proximal femoral BMD analysis. Initially, the body weight and blood glucose showed no significant differences $(\mathrm{P}>0.05)$ in both groups of rats before STZ injection. After 6 weeks, the diabetic rats had a significantly higher blood glucose and lower body weight when compared with the control group rats $(\mathrm{P}<0.01)$. Compared with non-diabetic control rats, diabetic rats showed a decrease in femoral BMD (-15.4 $\pm 2.3 \%$ vs. control, $\mathrm{P}<0.05$, Fig. 1).

$H \& E$ staining and immunohistochemistry assay. Osteoblasts were identified by a central lucency, eccentric nucleus and basophilic cytoplasmic stain. Osteoclasts were identified as single or multi-nucleated cells demonstrating foamy cytoplasm. Six weeks after inducing diabetes, the proximal femur H\&E staining demonstrated a greater number of osteoclasts that clumped together, reduced cortical bone and a deteriorated bone micro-architecture in diabetic rats. The overall growth plate architecture was dominated by hypertrophic chondrocytes, with fewer proliferative and chondroblastic cells as compared with control rats. The femur of control rats showed a normal shape with intact cartilage, qualitatively 
Table I. Comparison of C/EBP-homologous protein (CHOP)-positive cells and optical density in both groups (mean \pm SD).

\begin{tabular}{lcc}
\hline Group & No. of positive cells & Optical density \\
\hline Control rats & $8.3 \pm 2.1 / \mathrm{mm}^{2}$ & $102.4 \pm 12.1$ \\
Diabetic rats & $25.4 \pm 7.6 / \mathrm{mm}^{2 \mathrm{a}}$ & $180.3 \pm 17.4^{\mathrm{a}}$ \\
Osteoblasts in normal medium & $3.9 \pm 1.2 / \mathrm{mm}^{2}$ & $112.1 \pm 13.8$ \\
Osteoblasts in high-glucose medium & $43.7 \pm 11.4 / \mathrm{mm}^{2 \mathrm{~b}}$ & $176.1 \pm 15.3^{\mathrm{b}}$
\end{tabular}

${ }^{\mathrm{a}} \mathrm{P}<0.05$, vs. control rats, ${ }^{\mathrm{b}} \mathrm{P}<0.05$, vs. osteoblasts in normal medium.
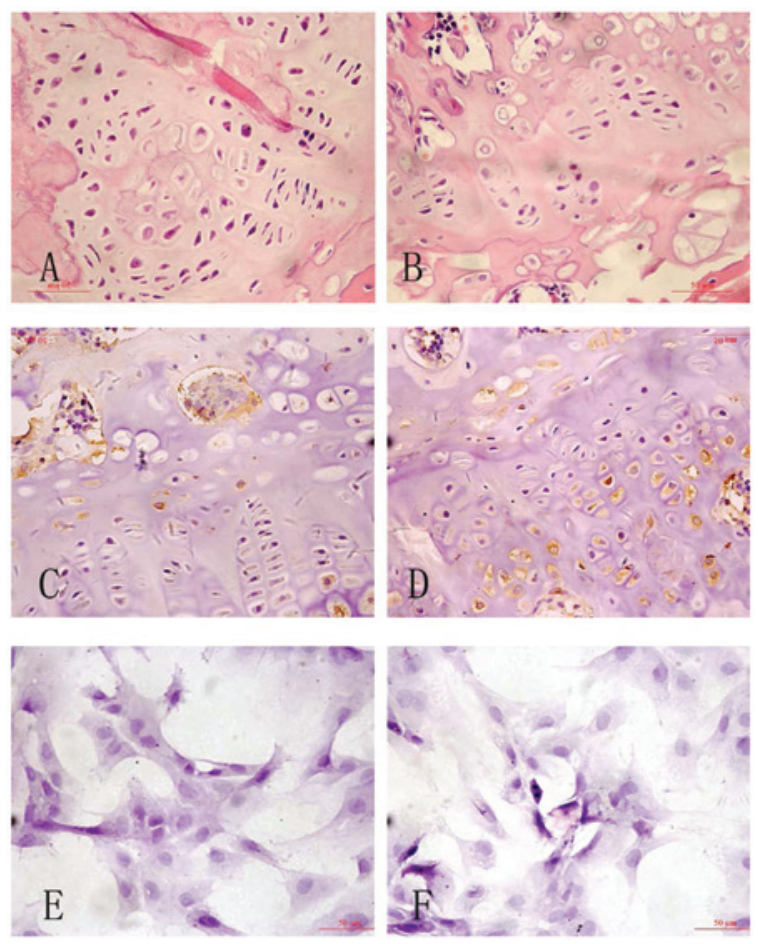

Figure 2. Hematoxylin and eosin (H\&E) staining and immunohistochemistry assay. H\&E staining of the proximal femur tissues; magnification, $\mathrm{x} 400$ (A-B) (A) A control rat. (B) A diabetic rat showed more osteoclasts that clumped together and reduced cortical bone. The overall growth plate architecture was dominated by hypertrophic chondrocytes, rare new bone formation with fewer proliferative and chondroblastic cells. Light photomicrographs of $\mathrm{C} / \mathrm{EBP}$-homologous protein (CHOP) immunohistochemical staining of the osteoblasts from proximal femur (C-D) and cultured osteoblasts (E-F) in the two groups. Cells that stained positive for CHOP showed buff-coloured granules with DAB staining. (C) A control rat showed few CHOP-positive osteoblasts. (D) A diabetic rat showed more CHOP-positive osteoblasts in the overall growth plate. (E) Control conditions (normal medium) showed few CHOP-positive osteoblasts. (F) Hyperglycemia condition showed more CHOP-positive osteoblasts.

equivalent cortical bone and a well-organized bone matrix composed of trabecular bone compared with the diabetic rats (Fig. 2).

CHOP immunoreactivity was visualized in a granular immunostain pattern in the nucleus. Caspase-12 immunohistochemistry staining positive cells with DAB staining, showed buff-colored granules in the cytoplasm. Quantitative analysis for the number and optical density of CHOP-positive cells with DAB immunostaining showed significant increases in osteo-
A
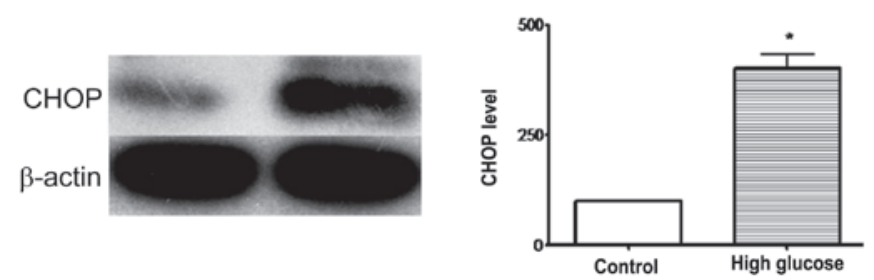

Figure 3. Western blot analysis for C/EBP-homologous protein (CHOP) in osteoblasts of both groups. (A) Expression levels of the CHOP protein in normal medium and hyperglycemia medium. Osteoblasts were assessed by western blot analysis and anti-beta-actin antibody (as a loading control). (B) The data are presented as the mean $\pm \mathrm{SD}$; ${ }^{*} \mathrm{P}<0.01$, vs. control rats.

blast cells in diabetic rats and under high-glucose medium in vitro $(\mathrm{P}<0.05$, Fig. 2 and Table $\mathrm{I})$.

The number of CHOP-positive cells and the optical density was significantly increased in the rats of the diabetic group $(\mathrm{P}<0.05)$. Similarly, the number of positive cells and the optical density was upregulated in the osteoblasts of highglucose medium compared with that of the normal medium group $(\mathrm{P}<0.05)$.

Western blot analysis. CHOP proteins in osteoblast cells were detected as single bands migrating $\sim 27 \mathrm{kDa}$. The densitometric analysis of the bands for $\mathrm{CHOP}$ revealed a significant increase in relative protein content $(451.4 \pm 32.6 \%)$ in highglucose medium osteoblasts when compared with those from the normal medium group (100.00\%; P<0.05, Fig. 3). This suggested that CHOP was activated in osteoblasts under high-glucose medium.

\section{Discussion}

DM is a group of pandemic debilitating metabolic diseases featuring chronic hyperglycemia which results from defective insulin secretion and/or insulin actions. Such chronic hyperglycemia typically elicits dysfunction and failure of various organs, particularly the eyes, kidneys, heart and nerves (12-15). In addition, DM has been found to be associated with metabolic bone diseases, osteoporosis and low-impact fractures (16). Patients with diabetes are at greater risk of fractures due to their low BMD. Lower BMD is explained by insulinopenia and hyperglycemia, which impair bone formation (17). Despite the discrepancies between BMD and fracture rates, clinical trials uniformly support the fact that new bone formation and bone 
microarchitecture and bone quality are altered in both types of diabetes $(18,19)$. In the present study, STZ-treated rats showed a decrease in femoral BMD compared with normal control rats. The femur of diabetic rats showed reduced cortical bone, deteriorated bone micro-architecture, a decrease in the trabecular width in the epiphysis and metaphysis and increased apoptosis of osteoblasts when compared with the control rats.

ER is the organelle where secretory and membrane proteins are synthesized and folded. In certain severe situations of ER stress, however, the protective mechanisms activated by the UPR are not sufficient to restore normal ER function and cells die by apoptosis (20). Hyperglycemia accumulates the misfolded proteins and induces alterations in $\mathrm{Ca}^{2+}$ homeostasis (21). The UPR in osteoblasts alleviate ER stress by upregulation of chaperones (such as BiP/GRP78) and degradation of misfolded proteins (22). However, under persistent hyperglycemia conditions, the UPR triggers apoptotic cell death (23). Microarray studies revealed that CHOP is one of the most highly inducible genes during ER stress and increases during ER stress during apoptosis (24). Overexpression of $\mathrm{CHOP}$ and microinjection of $\mathrm{CHOP}$ protein have been reported to play a key role in apoptosis by regulating the expression of proapoptotic proteins (25). Besides the possible mechanism that overexpression of CHOP in the bone microenvironment may impair the function of osteoblasts leading to osteopenia (26), CHOP may act as a dominant-negative inhibitor of C/EBP and prevent osteoblast differentiation (27). In the current diabetic osteoporosis rat model, diabetes significantly elevated the expression of CHOP in osteoblast cells. CHOP promotes osteoblast reversal of translational repression caused by the UPR to facilitate progress of apoptosis in vivo and in vitro. It may be hypothesized, therefore, that high blood sugar status, contributing to the high expression of CHOP, facilitates the progress of apoptosis, which upsets the osteoblast-osteoclast balance, leading to bone disorders and the development of diabetic osteoporosis. The relationship between CHOP expression and osteoclasts remains to be fully elucidated.

\section{Acknowledgements}

This study was supported by the National Natural Science Foundation of China (No. 30971124) and Zhejiang Provincial Natural Science Foundation of China (No. Y2090120).

\section{References}

1. Norris R and Parker M: Diabetes mellitus and hip fracture: A study of 5966 cases. Injury 42: 1313-1316, 2011.

2. Villafán-Bernal JR, Sánchez-Enríquez S and Muñoz-Valle JF: Molecular modulation of osteocalcin and its relevance in diabetes (Review). Int J Mol Med 28: 283-293, 2011.

3. Aubia J, Serrano S, Mariñoso L, Hojman L, Diez A, Lloveras J and Masramon J: Osteodystrophy of diabetics in chronic dialysis: a histomorphometric study. Calcif Tissue Int 42: 297-301, 1988.

4. He H, Liu R, Desta T, Leone C, Gerstenfeld LC and Graves DT: Diabetes causes decreased osteoclastogenesis, reduced bone formation, and enhanced apoptosis of osteoblastic cells in bacteria stimulated bone loss. Endocrinology 145: 447-452, 2004
5. Lecka-Czernik B: Bone loss in diabetes: use of antidiabetic thiazolidinediones and secondary osteoporosis. Curr Osteoporos Rep 8: 178-184, 2010.

6. Liu R, Bal HS, Desta T, et al: Diabetes enhances periodontal bone loss through enhanced resorption and diminished bone formation. J Dent Res 85: 510-514, 2006.

7. Martin TJ and Seeman E: Bone remodelling: its local regulation and the emergence of bone fragility. Best Pract Res Clin Endocrinol Metab 22: 701-722, 2008.

8. Van der Kallen CJ, van Greevenbroek MM, Stehouwer CD and Schalkwijk CG: Endoplasmic reticulum stress-induced apoptosis in the development of diabetes: is there a role for adipose tissue and liver? Apoptosis 14: 1424-1434, 2009.

9. Endo M, Oyadomari S, Suga M, Mori M and Gotoh T: The ER stress pathway involving CHOP is activated in the lungs of LPS-treated mice. J Biochem 138: 501-507, 2005.

10. Zinszner H, Kuroda M, Wang X, et al: $\mathrm{CHOP}$ is implicated in programmed cell death in response to impaired function of the endoplasmic reticulum. Genes Dev 12: 982-995, 1998.

11. Liu G, Sun Y, Li Z, Song T, Wang H, Zhang Y and Ge Z: Apoptosis induced by endoplasmic reticulum stress involved in diabetic kidney disease. Biochem Biophys Res Commun 370: 651-656, 2008.

12. Xie XW, Xu L, Jonas JB and Wang YX: Prevalence of diabetic retinopathy among subjects with known diabetes in China: the Beijing Eye Study. Eur J Ophthalmol 19: 91-99, 2009.

13. Senior PA: Diabetic nephropathy, chronic kidney disease and metabolic syndrome in Type 2 diabetes: answers or more questions? Diabet Med 25: 1377-1379, 2008.

14. Zhang XM, Shen F, Xv ZY, Yan ZY and Han S: Expression changes of thrombospondin-1 and neuropeptide $Y$ in myocardium of STZ-induced rats. Int J Cardiol 105: 192-197, 2005.

15. Zhou J, Wang L, Ling S and Zhang X: Expression changes of growth-associated protein-43 (GAP-43) and mitogen-activated protein kinase phosphatase-1 (MKP-1) and in hippocampus of streptozotocin-induced diabetic cognitive impairment rats. Exp Neurol 206: 201-208, 2007.

16. Kennedy RL, Henry J, Chapman AJ, Nayar R, Grant P and Morris AD: Accidents in patients with insulin-treated diabetes: increased risk of low-impact falls but not motor vehicle crashes - a prospective register-based study. J Trauma 52: 660-666, 2002.

17. Follak N, Kloting I and Merk H: Influence of diabetic metabolic state on fracture healing in spontaneously diabetic rats. Diabetes Metab Res Rev 21: 288-296, 2005.

18. Abbassy MA, Watari I and Soma K: The effect of diabetes mellitus on rat mandibular bone formation and microarchitecture. Eur J Oral Sci 118: 364-369, 2010.

19. Yamaguchi T, Yamamoto M, Kanazawa I, et al: Quantitative ultrasound and vertebral fractures in patients with type 2 diabetes. J Bone Miner Metab 29: 626-632, 2011.

20. Rasheva VI and Domingos PM: Cellular responses to endoplasmic reticulum stress and apoptosis. Apoptosis 14: 996-1007, 2009.

21. Wang S and Kaufman RJ: The impact of the unfolded protein response on human disease. J Cell Biol 197: 857-867, 2012.

22. Schröder M: Endoplasmic reticulum stress responses. Cell Mol Life Sci 65: 862-894, 2008.

23. Karunakaran U, Kim HJ, Kim JY and Lee IK: Guards and culprits in the endoplasmic reticulum: glucolipotoxicity and beta-cell failure in type II diabetes. Exp Diabetes Res 2012: 639762, 2012.

24. Cheng WP, Hung HF, Wang BW and Shyu KG: The molecular regulation of GADD153 in apoptosis of cultured vascular smooth muscle cells by cyclic mechanical stretch. Cardiovasc Res 77: $551-559,2008$.

25. Zhang X, He D, Xu L and Ling S: Protective effect of tanshinone IIA on rat kidneys during hypothermic preservation. Mol Med Report 5: 405-409, 2012.

26. Shirakawa K, Maeda S, Gotoh T, et al: CCAAT/enhancerbinding protein homologous protein (CHOP) regulates osteoblast differentiation. Mol Cell Biol 26: 6105-6116, 2006.

27. Pereira RC, Stadmeyer LE, Smith DL, et al: CCAAT/Enhancerbinding protein homologous protein (CHOP) decreases bone formation and causes osteopenia. Bone 40: 619-626, 2007. 